\title{
Using Prior Parameter Knowledge in Model-Based Design of Experiments for
}

\section{Pharmaceutical Production}

\author{
Ali Shahmohammadi a; Kimberley B. McAuley ${ }^{\text {b* }}$
}

McKetta Department of Chemical Engineering, University of Texas at Austin, Austin, TX 78712

b Department of Chemical Engineering, Queen’s University, Kingston, ON K7L 3N6, Canada

\section{ABSTRACT}

Sequential model-based design of experiments (MBDoE) uses information from previous experiments to select new experimental conditions. Computation of MBDoE objective functions can be impossible due to a non-invertible Fisher Information Matrix (FIM). Previously, we evaluated a leaveout (LO) approach that designed experiments by removing problematic model parameters from the design process. Unfortunately, the LO approach can be computationally expensive due to its iterative nature. In this study, we propose a simplified Bayesian approach that makes the FIM invertible by accounting for prior parameter information. We compare the proposed simplified Bayesian approach to the LO approach sequential A-optimal design. Results from a pharmaceutical case study show that the proposed

\section{*Corresponding Author: kim.mcauley@queensu.ca}

This article has been accepted for publication and undergone full peer review but has not been through the copyediting, typesetting, pagination and proofreading process which may lead to differences between this version and the Version of Record. Please cite this article as doi: 10.1002/aic.17021

(C) 2020 American Institute of Chemical Engineers

Received: Mar 24, 2020;Revised: Jul 04, 2020;Accepted: Aug 08, 2020

This article is protected by copyright. All rights reserved. 
approach is superior, on average, for design of experiments. We suggest that simplified Bayesian MBDoE should be combined with a subset-selection-based approach for parameter estimation. This combined methodology gave the best results on average for the case study.

Keywords: sequential model-based DoE, LO approach, simplified Bayesian approach, parameter estimation, mathematical modeling 


\section{INTRODUCTION}

Mathematical models are used in chemical and pharmaceutical industries for analysis, design and control of chemical processes and for maximizing product quality and profit. ${ }^{1,2}$ Especially in pharmaceutical industries, models are important for Quality by Design and development of continuous manufacturing processes, which are becoming more widespread. ${ }^{3-5}$ Mathematical models for pharmaceutical product development can be either empirical or mechanistic. ${ }^{5-7}$ Although empirical models are commonly used for pharmaceutical processes, they cannot reliably predict the system behavior outside the range of operating conditions used for model development. ${ }^{8}$ Therefore, fundamental models, based on underlying chemistry and physics, are preferred. ${ }^{9}$ These models usually contain unknown parameters that require estimation using experimental data. ${ }^{10}$ To obtain informative data, it is advantageous to carefully plan the experiments aimed at parameter estimation using design of experiment (DoE) techniques. ${ }^{11}$ As shown in Table 1, optimal model-based design-of-experiments (MBDoE) techniques select experiments to minimize uncertainties in parameters estimates or model predictions. ${ }^{12-}$

${ }^{14}$ MBDoE techniques are effective because they account for the structure of the model as well as parameter and measurement uncertainties when selecting new run conditions. ${ }^{13,15}$ Other benefits of ivinDoE techniques, compared to traditional full-factorial and fractional-factorial designs, are that they can be readily used to design any number of experiments, e.g., one, three or seven experiments, depending on available resources for experimentation. ${ }^{15,16}$ MBDoE techniques have been developed to satisfy a variety of objectives including minimizing total variances of parameter estimates, ${ }^{11,16-18}$ minimizing the average variance of model predictions, ${ }^{12,19,20}$ and designing experiments for model discrimination. ${ }^{21,22}$ 
Table 1 shows several MBDoE objective functions that have been used for development of chemical and pharmaceutical production models when measurement errors are assumed to be normally distributed. ${ }^{15,16,18}{ }^{18}$ When designing experiments modelers might be interested in accurate estimates or accurate model predictions. Table 1 shows MBDOE criteria that focus on parameter uncertainty (i.e., A-, D- and E- optimal designs) as well as criteria concerned with the propagation of parameter uncertainties into prediction uncertainties (i.e., G- and V-optimal designs). If modelers are interested in obtaining accurate parameter estimates for their model, A-, D- or E- optimal designs can be selected. ${ }^{15,16,18}$ Alternatively, G- and V-optimal designs focus on obtaining accurate model predictions at specified operating conditions of interest to the modeler. ${ }^{20,23-25}$ All of these MBDoE techniques in Table 1 require computation of the inverse of the Fisher Information Matrix (FIM) when selecting experimental settings. ${ }^{17,25,26}$ The FIM carries information about how changes in parameter values can affect the model predictions and is therefore crucial for both MBDoE calculations and parameter inference. ${ }^{27}$ For nonlinear models, which are common in chemical and pharmaceutical applications, computation of the FIM requires linearizing the model around some nominal parameter values. ${ }^{18,28}$ If these nominal parameter values are significantly different from the corresponding true values, the selected MBDoE settings may lead to csperimental data that are not very informative. ${ }^{18,28}$ Sequential design approaches are appealing because they enable updating of the parameter values, as well as the experimental strategy, as more data become available. ${ }^{29}$ Using sequential experimental designs, valuable information from old experimental data can be used, which might have been collected for other objectives than model development. ${ }^{30,31}$ It is also possible to use MBDoE methods to design a number of new experiments in parallel. A modeler may 
choose to update the parameters and perform new MBDoE calculations after a portion of the proposed new experiments is conducted.

Computation of the objective functions for sequential MBDoE is problematic if the FIM is noninvertible or ill-conditioned. A singular FIM can be due to the model structure (a structural identifiability problem) or to a lack of informative experimental data for parameter estimation (a practical identifiability problem, also known as an estimability problem). ${ }^{22,32,33}$ A model is structurally identifiable if unique estimates for the parameters could be obtained using sufficient noise-free observations from well-designed experiments. ${ }^{34}$ These types of identifiability problems can be addressed by reparametrizing the model or by changing the model's structure. The model parameters are recognized as estimable (practically identifiable) if unique parameter estimates can be obtained using the available experimental data. ${ }^{33}$ Estimability problems can be addressed by conducting additional informative experiments or by simplifying the model. ${ }^{33}$ In chemical, biochemical and pharmacological systems, models often contain a large number of kinetic and transport parameters (e.g., 10-80 parameters) which may result in noninvertible/ill-conditioned FIMs. ${ }^{35-39}$ To avoid this problem, several approaches have been considered during sequential MBDoE calculations including parameter-subset selection, ${ }^{14,40,41}$ pseudoinverse wuthods, ${ }^{25,42}$ Tikhonov regularization, ${ }^{43-46}$ and Bayesian approaches. ${ }^{13,47,48}$

Table 1. Optimality criteria for model-based design of experiments

The parameter-subset-selection approach uses a model-reduction perspective. ${ }^{41,49,50}$ In one methodology, parameters are ranked from most-estimable to least-estimable so that problematic (lowranked) parameters can be recognized and fixed at their nominal values. ${ }^{41,51}$ In this way, experiments can 
be designed using a well-conditioned reduced FIM that ignores problematic parameters. Alternatively, pseudoinverse methods approximate the inverse of the FIM (e.g., using the Moore-Penrose pseudoinverse) during MBDoE calculations. ${ }^{25,42,52}$ In Tikhonov regularization, a penalty is added to diagonal elements of the FIM to make it invertible. ${ }^{40,44-46}$ Bayesian MBDoE using linear models results in Tikhonov penalties that account for prior knowledge about parameters. However, for nonlinear models, the situation can be considerably more complex, depending on how the nonlinearity is treated. ${ }^{13,47,48}$ There is little information in the literature regarding which approach is most effective. In two previous articles, we considered pharmaceutical case studies involving noninvertible FIMs. Two different approaches were compared: i) a subset-selection-based approach that leaves out problematic parameters (LO approach) and ii) a simpler approach that uses a Moore-Penrose pseudoinverse in place of $\mathbf{F I M}^{\mathbf{- 1}}$ (PI approach). ${ }^{25,52}$ These case studies suggest that the LO approach is often superior to the PI approach for designing both A- and V-optimal experiments. ${ }^{25,52} \mathrm{~A}$ shortcoming of the LO approach is that it can be complicated and computationally expensive due to changes in the subset of parameters that is left out during MBDoE calculations. This complication motivates us to find a more convenient approach to deal with singular FIMs during MBDoE.

The focus of the current study is on a simplified Bayesian approach for dealing with singular/illconditioned FIMs during MBDoE. Bayesian approaches have been used in several past MBDoE studies for chemical and biochemical systems. ${ }^{53-55}$ The main benefit of the simplified Bayesian MBDoE framework is that it accounts explicitly for prior knowledge about plausible values of the model parameters. ${ }^{13}$ However, many researchers raise concerns about the use of Bayesian approaches in practical 
engineering systems. ${ }^{13,56,57}$ Disadvantages of the Bayesian approach include uncertainty about the reliability of assumptions made when specifying prior information. ${ }^{55,56,58}$ Undesirable computational complexity can also arise, depending on the assumptions that are made. As a result, Bayesian MBDoE has not enjoyed widespread applications in chemical process modeling. In this study, we developed a new Tikhonov-based approach for MBDoE to address the problem of ill-conditioned/singular FIMs. The Tikhonov weightings are specified by using Bayesian arguments based on the modeler's prior knowledge about plausible values of the parameters. This approach has never been used before and results in a simplified Bayesian MBDoE objective function that can be used during sequential MBDoE when the FIM is noninvertible.

The objective of the current article is to formulate and test a simplified Bayesian MBDoE approach that is readily usable by model developers. In Chemical and biochemical systems, engineers who develop fundamental models have some prior knowledge (at least about the order of magnitude) of many of their model parameters. It is important to use this prior knowledge effectively when the data lack sufficient information to estimate all of the parameters. The proposed simplified Bayesian approach is devised to use this prior knowledge when designing new experiments. The effectiveness of the proposed Bayesian approach is compared to that of the LO approach for designing A-optimal experiments when the FIM is noninvertible. While Bayesian MBDoE explicitly uses prior parameter information for designing new experiments, the LO MBDoE approach implicitly incorporates prior parameter information using appropriate scaling factors. We use the pharmaceutical case study of Domagalski et al., (2015), which is of interest to our industrial sponsor. ${ }^{6,25}$ The associated dynamic model uses Michaelis-Menten kinetics 
and enzyme-catalyzed reactions to describe the production of a pharmaceutical agent. ${ }^{59}$ Note that, the main performance criterion used for assessing the effectiveness of the LO and Bayesian experimental design is considered as sum of squared deviations between the resulting parameter estimates and the true values used to generate the simulated data. This performance criterion is consistent with the A-optimal MBDoE criterion which is the main reasons we choose A-optimal design criterion in this study.

The remainder of this article is organized as follows. First, background on the FIM and sequential A-optimal design is presented. Next, details of the Bayesian and LO approaches for parameter estimation and experimental design are presented. A simple Bayesian approach is proposed and a pharmaceutical case study is presented. Results obtained using Monte Carlo (MC) simulations are provided, revealing that the proposed Bayesian approach is superior to the LO approach for this case study.

\section{BACKGROUND INFORMATION}

\section{Sequential MBDoE for nonlinear models}

Consider the nonlinear model:

$$
\mathbf{Y}=\mathbf{g}(\mathbf{d}, \boldsymbol{\theta})+\boldsymbol{\varepsilon}
$$

where $\mathbf{Y} \in \mathbf{R}^{N}$ is a vector of stacked measured responses, $\mathbf{g}$ is the solution of equations that describe the sy tem, $\mathbf{d} \in \mathbf{R}^{r \times D}$ is a matrix of experimental settings (for $r$ runs with $D$ decision variables specified for each), $\boldsymbol{\theta} \in \mathbf{R}^{p}$ is the vector of model parameters and $\boldsymbol{\varepsilon} \in \mathbf{R}^{N}$ is a vector of a measurement noise with diagonal covariance matrix $\boldsymbol{\Sigma}_{\mathbf{y}} \in \mathbf{R}^{N \times N}$. For dynamic multi-response models with $n$ sample times per run and $v$ response variables, the total number of data values is $N=n v r$. The FIM is computed using a parametric sensitivity matrix $\mathbf{S} \in \mathbf{R}^{N \times p}$ with elements: 


$$
S_{i j}=\left.\frac{\partial \mathrm{g}(\mathbf{d}, \boldsymbol{\theta})}{\partial \theta_{j}}\right|_{\widehat{\theta}_{k \neq j}}
$$

computed by linearizing the model around the best currently-available parameter values: ${ }^{60}$

The elements of $\boldsymbol{S}$ should be scaled using parameter uncertainties $s_{\theta_{j}}$ and measurement uncertainties $s_{y_{i}}$ to reflect the modeler's prior knowledge: ${ }^{61}$

$$
Z_{i j}=S_{i j} \frac{s_{\theta_{j}}}{s_{y_{i}}}
$$

resulting in a scaled sensitivity matrix $\mathbf{Z}$. The $\mathbf{F I M}$ is related to $\mathbf{Z}$ by:

$$
\mathbf{F I M}=\mathbf{Z}^{T} \mathbf{Z}
$$

When performing sequential MBDoE calculations, $\mathbf{Z}$ contains two parts: ${ }^{25,52}$

$$
\mathbf{Z}=\left[\begin{array}{c}
\mathbf{Z}_{\text {old }} \\
\mathbf{Z}_{\text {new }}
\end{array}\right]
$$

where $\mathbf{Z}_{\text {old }}$ corresponds to experimental settings and data from old experiments. The elements of $\mathbf{Z}_{\text {old }}$ are fixed during sequential MBDoE and elements of $\mathbf{Z}_{\text {new }}$ are determined by the optimizer. After each sequential design, elements of $\mathbf{Z}_{\text {old }}$ are updated based on the new parameter values and the number of rows in $\mathbf{Z}_{\mathbf{o l d}}$ increases due to the recent experiments.

\section{Parameter estimation with a noninvertible FIM}

When estimating parameters, the FIM should be invertible, otherwise unique estimates for the parameters cannot be obtained. ${ }^{17,40}$ Several regularization approaches have been used to overcome this problem. ${ }^{44,45,62}$ One popular approach is to estimate a subset of the model parameters that are estimable, with the remaining parameters fixed at nominal values. ${ }^{26,51,63}$ Table 2 shows computational steps for a commonly used orthogonalization-based approach that ranks parameters from the most-estimable so 
problematic (unranked) parameters that lead to a noninvertible FIM can be determined. ${ }^{51,61}$ The ranking starts by computing the magnitude of each column of the scaled sensitivity matrix $\mathbf{Z}$ (Step 1). The parameter corresponding to the column with the highest magnitude is selected as the most-estimable parameter (Step 2). The columns of $\mathbf{Z}$ are then regressed onto columns of $\mathbf{X}_{k}$, a matrix that contains columns from $\mathbf{Z}$ that correspond to the ranked parameters (Step 3). Residual matrix $\mathbf{R}_{k}$ is then computed to remove correlation between columns for the unranked parameters and columns for the parameters that have already been ranked (Step 4). The next-most-estimable parameter is the one with the largest magnitude among columns of $\mathbf{R}_{\boldsymbol{k}}$. In Step 5, the column corresponding to the next-most-estimable parameter is selected from the original $\mathbf{Z}$ matrix and included in $\mathbf{X}_{k}$, resulting in matrix $\mathbf{X}_{k+1}$. Steps two to five are repeated to produce a ranked list with up to $p$ parameters. The ranking stops when all of the parameters are ranked or at the iteration where $\mathbf{X}_{k}^{T} \mathbf{X}_{k}$ (the reduced FIM) becomes noninvertible. The remaining unranked parameters are categorized as problematic. These unranked parameters may be influential but are not selected for estimation because their uncertainty-scaling factors indicate that they are already well known by the modeler. Alternatively, they may be less-important parameters that have little or no influence on predictions of the available data. As such, orthogonalization focuses the limited available information on parameters whose values are poorly known and on parameters that are most influential compared to their initial uncertainty levels. ${ }^{33,51}$ Using this orthogonalization-based ranking approach prior to parameter estimation helps to avoid numerical problems that would arise due to a noninvertible FIM. Note that the methodology provided in Table 2 will be used in this case study when 
designing experiments and using the LO approach, which a comparator method used for testing the effectiveness of the proposed simplified Bayesian method.

Table 2. Orthogonalization algorithm ${ }^{51,61}$

Unfortunately, estimating the complete set of ranked parameters can lead to overfitting and poor model predictions. ${ }^{64,65}$ To solve this problem, Wu et al. (2011) developed a mean-squared-error-based criterion, which has been used to select an appropriate number of ranked parameters for estimation in a variety of fundamental modeling studies. ${ }^{64}$ Orthogonalization and Wu's criterion are used in the LO approach for parameter estimation in the current study.

An alternative approach to parameter-subset selection is to use Bayesian parameter estimation. ${ }^{66-}$ 68 Sometimes a modeler may have prior knowledge about parameters (i.e., may be willing to make assumptions about reasonable parameter values and their uncertainties). Assuming that there exists a set of prior estimates or initial guesses, $\widehat{\boldsymbol{\theta}}_{0}$, for the parameter values and assumed prior variance/covariance matrix $\boldsymbol{\Sigma}_{0}$, then the objective function for Bayesian weighted least-squares parameter estimation is: ${ }^{13,58,68}$

$$
\widehat{\boldsymbol{\theta}}_{B}=\arg \min _{\boldsymbol{\theta}}\left\{\sum_{i=1}^{N} \frac{\left(y_{i}-g\left(x_{i}, \boldsymbol{\theta}\right)\right)^{2}}{s_{y_{i}}^{2}}+\left(\boldsymbol{\theta}-\widehat{\boldsymbol{\theta}}_{0}\right)^{T} \boldsymbol{\Sigma}_{0}^{-1}\left(\boldsymbol{\theta}-\widehat{\boldsymbol{\theta}}_{0}\right)\right\}
$$

equation (6) $\boldsymbol{\Sigma}_{0}$ is a user-specified $p \times p$ matrix, which is usually diagonal. Based on the objective function in equation (6), an unscaled FIM, FIM $\mathbf{M}_{\mathbf{u s}}^{\mathbf{B}}$, for Bayesian parameter estimation has the following form: $:^{31,69-71}$

$$
\operatorname{FIM}_{\mathrm{uS}}^{\mathrm{B}}=\mathrm{S}^{\mathrm{T}} \Sigma_{\mathrm{y}}^{-1} S+\Sigma_{0}^{-1}
$$


where $\boldsymbol{\Sigma}_{\mathbf{y}}^{-\mathbf{1}}$ is a diagonal matrix of user-specified measurement variances. In equation (7), the first term on the right-hand side accounts for information contained in the data and the second term accounts for prior information about the parameters, specified by the modeler.

The advantage of using equations (6) and (7) is that they account for the modeler's prior knowledge about parameter values and their uncertainties when estimating parameters and computing confidence intervals. In addition, the prior information improves the conditioning of the FIM (by increasing the size of the diagonal elements as in Tikhonov regularization) helping to solve problems associated with noninvertible FIMs. ${ }^{72,73}$ One concern that has often been expressed concerning Bayesian estimation is that it results in the estimation of all the parameters, even those where the data contain little or no information about them. A second concern is that modelers have difficulties in specifying prior uncertainty levels for poorly-known parameters. They worry that misleading prior information could lead to unreliable parameter estimates and associated decisions. In the current study, the objective function in equation (6) will be used for Bayesian parameter estimation and the results will be compared with those from a parameter-subset-selection approach.

\section{Bayesian MBDoE}

As an alternative to the LO approach for MBDoE, which was investigated in our previous study, Bayesian MBDoE methods can be used. Bayesian methods are powerful they incorporate prior information in the design procedure. ${ }^{53,74,75}$ The Bayesian experimental design framework uses "utility functions" that measure the anticipated information gain due to the data. ${ }^{76}$ In this way, Bayesian MBDoE 
settings are those that maximize the expected value of the utility function given the model and the prior information. ${ }^{13,76,77}$ Lindley (1995) defined the expected utility function $U(\mathbf{d})$ :

$$
U(\mathbf{d})=\iint u(\mathbf{d} \mid \boldsymbol{\theta}, \mathbf{Y}) P(\boldsymbol{\theta}, \mathbf{Y} \mid \mathbf{d}) d \boldsymbol{\theta} d \mathbf{Y}
$$

where $u(\mathbf{d} \mid \boldsymbol{\theta}, \mathbf{Y})$ is a utility function, given the model parameters, $\boldsymbol{\theta}$, and the experimental outcome $\mathbf{Y}$, obtained using experimental settings $\mathbf{d}$. An important step in Bayesian MBDoE is to select an appropriate utility function, $u(\mathbf{d} \mid \boldsymbol{\theta}, \mathbf{Y})$, that reflects the goal of the experimentation. For example, one may decide to maximize the Kullback-Leibler distance between the prior and posterior probability distribution for the parameters: $:^{13,57,74,76}$

$$
U_{1}(\mathbf{d})=\iint \ln [P(\boldsymbol{\theta} \mid \mathbf{Y}, \mathbf{d})] P(\boldsymbol{\theta}, \mathbf{Y} \mid \mathbf{d}) d \boldsymbol{\theta} d \mathbf{Y}
$$

Assuming a Gaussian posterior distribution for the parameters, objective function (9) is Bayesian Doptimal design. ${ }^{13,78}$ Chaloner and Verdinelli (1995) considered a linear regression model of the form $\mathbf{Y}=$ $\mathbf{X} \boldsymbol{\theta}+\boldsymbol{\varepsilon}$, where $\mathbf{X} \in \mathbf{R}^{N \times p}$ is the design matrix for the linear regression model. ${ }^{13}$ They showed that maximizing the objective function in equation (9) corresponds to minimizing the following commonlyused Bayesian D-optimal objective function: ${ }^{13}$

$$
J_{B D}=\operatorname{det}\left\{\left[\sigma^{-2} \mathbf{X}^{\mathbf{T}} \mathbf{X}+\mathbf{\Sigma}_{\mathbf{0}}^{-1}\right]^{-1}\right\}
$$

where $\sigma^{-2} \mathbf{X}^{\mathbf{T}} \mathbf{X}$ is the $\mathbf{F I M}$ for a single response linear regression model with measurement variance $\sigma^{2}$ and $\boldsymbol{\Sigma}_{\mathbf{0}}$ is the assumed prior variance-covariance matrix for the parameters. Note that the $\mathbf{X}$ matrix corresponds to the unscaled sensitivity matrix (see equation (2)) for the linear regression model.

Similarly, Chaloner and Verdinelli (1995) showed that the Bayesian A-optimal objective function for their linear regression model: ${ }^{13}$ 


$$
J_{B A}=\operatorname{trace}\left\{\left[\sigma^{-2} \mathbf{X}^{\mathbf{T}} \mathbf{X}+\mathbf{\Sigma}_{\mathbf{0}}^{-\mathbf{1}}\right]^{-1}\right\}
$$

can be obtained from the expected utility function:

$$
U_{2}(\mathbf{d})=\iint\left(\boldsymbol{\theta}-\widehat{\boldsymbol{\theta}}_{0}\right)^{T} \Sigma_{0}^{-1}\left(\boldsymbol{\theta}-\widehat{\boldsymbol{\theta}}_{0}\right) P(\boldsymbol{\theta}, \mathbf{Y} \mid \mathbf{d}) d \boldsymbol{\theta} d \mathbf{Y}
$$

Objective functions (10) and (11) are appropriate for situations where the model is linear and single response with Gaussian priors specified for the parameters. For nonlinear models, analytical solutions for the double integrals in equations (9) and (12) are far more difficult to obtain. As such, simplifying assumptions have been used to develop approximate Bayesian D- and A- optimal objective functions for use in nonlinear mathematical model development. ${ }^{13,76}$ Two common approaches for simplifying these objective functions are i) a sampling approach which uses numerical integration ${ }^{72,77}$ and ii) an asymptotic approach which uses the linearized form of the model when designing new experiments. ${ }^{77,79}$ The sampling approach becomes computationally expensive for models with a large number of parameters or for systems with large numbers of measurements. ${ }^{13,55,72}$ As such, asymptotic approach is more convenient and practical for larger-scale nonlinear models. ${ }^{13,80}$

Considering that a nonlinear model can be linearized around its parameter initial guesses, the expression $\sigma^{-2} \mathbf{X}^{\mathbf{T}} \mathbf{X}+\boldsymbol{\Sigma}_{\mathbf{0}}^{-1}$ in equations (10) and (11) is a special case of the right-hand side for equation (8). As such, we can specify D-optimal MBDoE objective functions for multi-response nonlinear models as: ${ }^{13,73}$

$$
J_{B D}=\operatorname{det}\left\{\left(\mathbf{S}^{\mathrm{T}} \boldsymbol{\Sigma}_{\mathbf{y}}^{-\mathbf{1}} \mathbf{S}+\boldsymbol{\Sigma}_{\mathbf{0}}^{-\mathbf{1}}\right)^{-1}\right\}
$$

Objective function (13) is sometimes called a local objective function, because, the $\mathbf{S}$ matrix is obtained by linearizing the model around the initial or best available parameter values. ${ }^{17,18,73,74}$ Box and 
Lucas (1959) explained the prior variance-covariance matrix $\boldsymbol{\Sigma}_{\mathbf{0}}^{\mathbf{- 1}}$ is often neglected because modelers may not have informative prior information about parameter values. In this situation, a non-Bayesian Doptimal MBDoE is conducted (see objective function (1.2) in Table 1). However, a benefit of considering $\boldsymbol{\Sigma}_{\mathbf{0}}^{\mathbf{- 1}}$ in the objective function (13) is that this term can improve the conditioning of the FIM, while incorporating any prior information about plausable parameters values. The prior information becomes less important as additional experiments are performed and more data become available. Similar to Bayesian D-optimal experiments, Bayesian A- optimal experiments can be obtained by minimizing:

$$
J_{B A}=\operatorname{trace}\left\{\left(\mathbf{S}^{\mathbf{T}} \boldsymbol{\Sigma}_{\mathbf{y}}^{-1} \mathbf{S}+\boldsymbol{\Sigma}_{\mathbf{0}}^{-\mathbf{1}}\right)^{-1}\right\}
$$

\section{Proposed simplified Bayesian MBDoE}

It is convenient to scale the sensitivity matrix $\mathbf{S}$ to produce scaled sensitivity matrix $\mathbf{Z}$, i.e., by multiplying both sides of $\mathbf{F I M}_{\mathrm{us}}^{\mathrm{B}}$ by $\boldsymbol{\Sigma}_{\mathbf{0}}$ giving:

$$
\operatorname{FIM}^{\mathrm{B}}=\mathbf{S}^{\mathrm{T}} \boldsymbol{\Sigma}_{\mathbf{0}} \boldsymbol{\Sigma}_{\mathbf{y}}^{-1} \mathrm{~S}+\boldsymbol{\Sigma}_{\mathbf{0}} \boldsymbol{\Sigma}_{\mathbf{0}}^{-1}=\mathbf{Z}^{\mathrm{T}} \mathrm{Z}+\mathbf{I}_{\mathbf{p} \times \mathbf{p}}
$$

where $\mathbf{Z}$ is the scaled sensitivity matrix defined in equation (3) and $\mathbf{I}_{\mathbf{p} \times \mathbf{p}}$ is a $p \times p$ identity matrix. Note that the scaling parameters $s_{\theta j}$ in equation (3) is selected to be prior standard deviation for $j$ th parameter specified by the modeler which is the square root of $j$ th diagonal element of $\boldsymbol{\Sigma}_{\mathbf{0}}$. Also, $s_{y_{i}}$ is the measurement standard deviation corresponding to the square roots of the diagonal elements of $\boldsymbol{\Sigma}_{\boldsymbol{y}}$. In equation (15), the $\mathbf{Z}^{\mathrm{T}} \mathbf{Z}$ term on the right-hand-side accounts for information in the data and the identity matrix accounts for prior information specified by the modeler. The benefit of this new equation is that it incorporates prior knowledge about parameters using the same scaling factors that are used for scaling the sensitivities during parameter-subset selection. Because this scaling leads to an identity matrix for the 
right-most term in equation (15), this new expression will be simple to use, especially when the FIM is not invertible. Therefore, simplified Bayesian A- optimal experiments can be obtained by minimizing:

$$
J_{B-A}^{*}=\operatorname{trace}\left(\mathbf{F I M}^{\mathrm{B}^{-1}}\right)=\operatorname{trace}\left(\left(\mathbf{Z}^{\mathrm{T}} \mathbf{Z}+\mathbf{I}_{\mathbf{p} \times \mathbf{p}}\right)^{-1}\right)
$$

The benefit of using $\mathbf{F I M}^{\mathrm{B}^{-1}}$ in place of $\mathbf{F I M}^{-\mathbf{1}}$ is that, it can easily remove the problem of a singular FIM by taking into account prior knowledge about reasonable parameter values. As in other forms of Tikhonov regularization, positive numbers are added to the diagonal of the FIM thereby improving conditioning. Objective functions (16) is a simplified Bayesian MBDoE which can be readily used by the modelers who have scaled their sensitivity matrices for the purpose of orthogonalization and parameter-subset selection. The only additional assumption that the modeler is required to make is that the prior scaling information for each parameter corresponds to a Gaussian distribution.

In the current article, the proposed simplified Bayesian approach for MBDoE is compared with the previously-developed LO approach for MBDoE using a nonlinear case study involving the production of a pharmaceutical agent. We are interested in investigating whether it is better to formally incorporate prior knowledge about parameters to deal with a noninvertible FIM or it is better to remove the problematic parameters from the design. Investigations are also carried out on the robustness of the simplified Bayesian and LO approaches to the accuracy of the prior parameter information. Although the focus of this study is on the design of experiments, we also investigate the effectiveness of Bayesian and LO parameter estimation using the new data obtained following MBDoE. We are interested in whether it is better to use a weighted-least-squares Bayesian approach to estimate all of the parameters or it is better 
to estimate only a subset of the parameters via the LO approach. In addition, the computational requirements of the two approaches are compared.

\section{MICHAELIS-MENTEN CASE STUDY}

\section{Reaction scheme and dynamic model}

The case study considered in the current article uses a nonlinear kinetic model based on a

Michaelis-Menten batch reaction for the production of a pharmaceutical agent. Domagalski et al. (2015) used this case study to develop empirical models based on conventional DoE and response surface methodology. ${ }^{6}$ We used the same case study to develop and test the LO approach for V-optimal MBDoE in previous work. $^{25}$ The reaction starts with reagent SM1 reacting with catalyst D and generating intermediate SM1.D via reversible reaction (1) in Figure 1. Next, intermediate SM1.D reacts with reagent SM2 to make the product P and release the catalyst (i.e., reaction (2)). There is also a possibility of generating several impurities: SM2 can react with P to generate impurity I1, SM1 can be hydrolyzed to form impurity I2, D can be deactivated with water to make I3, and P can degrade to generate I4. The fundamental model describing the reqction scheme in Figure 1 is provided in Table S3 in the Sublementary Information.

In the study by Domagalski et al., 3 rounds of simulated experiments were performed. In each round, they conducted 16 fractional-factorial runs +4 center-point-runs (i.e., 20 experiments in each round and 60 overall). Table 3 shows Domagalski's center-point settings for their first round of experimentation.

We assume that data for the 4 replicated center-point runs are available for initial parameter estimation and construction of $\mathbf{Z}_{\mathbf{o l d}}$. Step-by-step computation of $\mathbf{Z}_{\mathbf{o l d}}$ using these runs is described in the 
Supplementary Information. The duration of each simulated batch experiment is $6.0 \mathrm{~h}$ with measurements taken every 45 minutes, resulting in sampling at 9 times including the initial time $t=0$. As a result, each run involves 36 measured values (i.e., 9 values each for $y_{S M 1}, y_{D}, y_{S M 2}$ and $y_{P}$ ).

Figure 1. Reaction scheme for Michaelis-Menten case study ${ }^{6}$

Table 3. Initial conditions for center-point batch reactor operation at $T=40^{\circ} \mathrm{C}$

Table 4 shows the true kinetic coefficients used by Domagalski et al. for generating simulated data. These parameter values were used in the current study to compute true kinetic and equilibrium coefficients via Arrhenius expressions:

$$
\begin{aligned}
& k_{i}(T)=k_{i, \text { ref }} \exp \left(-\frac{E_{a, i}}{R}\left(\frac{1}{T}-\frac{1}{T_{\text {ref }}}\right)\right) \\
& K_{e q}(T)=K_{\text {eq, ref }} \exp \left(-\frac{\Delta H_{1}}{R}\left(\frac{1}{T}-\frac{1}{T_{\text {ref }}}\right)\right)
\end{aligned}
$$

where $k_{i}$ is the $i$ th kinetic coefficient, $R$ is the universal gas constant, $T$ is the temperature in $\mathrm{K}$, and $T_{r e f}=$ $313.15 \mathrm{~K}=40{ }^{\circ} \mathrm{C}$ is a reference temperature. In equation (18), $K_{e q}$ is the equilibrium coefficient for reaction (1), and $\Delta H_{1}$ is the reaction enthalpy. Table 5 provides measurement noise variances used in this study for generating simulated data. ${ }^{25}$ Figure 2 shows one set of simulated old data generated using the values in Table 4 and Table 5. As shown in the simulated true response in Figure 2, consumption of catalyst D is initially very fast and then the catalyst gets released via reaction (2) as the product is formed.

Table 4. True values of the kinetic coefficients and equilibrium constant ${ }^{6}$

Table 5. Measurement variances used for generating simulated data 
The initial case study assumes that the system operates at $T=T_{r e f}=40{ }^{\circ} \mathrm{C}$, which results in seven parameters requiring estimation (i.e., $\boldsymbol{\theta}=\left[k_{1 f}, K_{e q}, k_{2}, k_{3}, k_{4}, k_{5}, k_{6}\right]^{T}$ ). These seven parameters lead to seven columns in the scaled sensitivity matrix $\mathbf{Z}$. Decision variables for the new experiments are initial concentrations for the reactants SM1, D, and SM2 (i.e., $\mathbf{d}=\left[C_{S M 1_{0}}, C_{D_{0}}, C_{S M 2_{0}}\right]^{T}$ ). Lower and upper bounds for these decision variables are provided in Table 6. Note that, the four approaches (i.e., LO-LO, Bayes-LO, LO-Bayes and Bayes-Bayes) are also considered in an expanded case study (reported in the Supplementary Information) with the temperature as an additional decision variable $\left(35^{\circ} \mathrm{C} \leq T \leq 45^{\circ} \mathrm{C}\right)$. As a result, the model is more complicated with 14 unknown parameters (including the activation energies and $\Delta H)$.

Figure 2. Simulated data (symbols) and noise-free concentration profiles (curves) obtained at $40{ }^{\circ} \mathrm{C}$ using experimental settings in Table 3, kinetic coefficients in Table 4 and measurement errors in Table 5: a) SM1; b) D; c) SM2; d) P.

In the initial case study, three new sequential experiments are designed one-at-a-time. In the first step, one A-optimal experiment is designed and the parameters are estimated using both the old and the new data. Next, these parameter estimates and all of the data obtained are used to design a second sequential experiment. Finally, a third experiment is designed using parameter estimates and data from previous steps. For comparison, three new experiments are designed all-at-once based on the old data in Figure 2 and corresponding parameter estimates.

Table 6. Lower and upper bounds for the decision variables 
Table 8 provides information about the user-specified prior parameter information used in three different Cases. The prior parameter guesses and corresponding standard deviations were used in the Bayesian objective function for parameter estimation (equation (6)). This prior information is also used in LO parameter estimation to obtain scaled sensitivity coefficients in $\mathbf{Z}$ (see equations (2) and (3)). As result, prior assumptions about parameters influence which parameters are estimated and which remained fixed at their initial values. The three cases described in Table 8 were used to investigate the influence of the prior parameter information on the quality of parameter estimates and experimental settings. For all three cases, parameter initial guess $\hat{\theta}_{j 0}$ were selected randomly from normal distributions with true mean $\theta_{j}$ and true standard deviation $s_{\theta_{j}}$ (see Table 4). In Case I, the modeler specifies prior information that is quite accurate (i.e., prior parameter standard deviations are 1/5 of the true value), whereas in Case II, the modeler is less certain about the initial parameter guesses. The selection rules in the third column of Table 8 for Cases I and II prevent random selection of unrealistic negative parameter values and parameter values more than 3 standard deviations from the true parameter values. Case III is used to investigate whether the Bayesian or $\mathrm{LO}$ approach to MBDOE and parameter estimation is more robust to misinformed prior information (i.e., when modelers mistakenly believe that they know more about the plausible parameter values than is warranted). In Case III, initial parameter guesses are further from the true values than the modeler believes. Note that, Cases I, II and III are different in the initial parameter guesses used for linearization and in the parameter scaling factor $s_{\theta_{j}}$ as shown in Table 7. We kept the measurement uncertainties $s_{y_{i}}$ similar for all three cases.

Table 7. Selection of parameter initial guesses from normal distributions 


\section{MONTE CARLO SIMULATION RESULTS AND DISCUSSION}

\section{Case I: Results when informative parameter initial guesses are used}

In this Case, 100 initial guesses for the seven parameters were used (as described in Table 8) along with 100 different simulated old datasets. We chose 100 sets of parameter initial guesses because we did not want the conclusions from our study to depend too much on any particular set of initial guesses. Using each set of initial guesses and the simulated old datasets, preliminary values of the model parameters were estimated using both Bayesian and LO approaches. All seven parameters were estimated using the Bayesian approach, whereas subsets of parameters were estimated using the LO approach, with remaining parameters fixed at their initial guesses. Using the LO approach, parameter $k_{2}$ was always ranked as the most-estimable parameter, followed by $K_{e q}, k_{1 f}$ and $k_{3}$ (using the ranking algorithm in Table 2). Parameters $k_{4}, k_{5}$ and $k_{6}$ were always left out of the ranked list. Using Wu's $r_{c c}$ criterion, the parameter subset $\boldsymbol{\theta}_{\text {sub }}=\left[k_{2}, K_{e q}\right]^{T}$ was selected for estimation in all 100 simulated old data sets. Parameters $k_{1 f}$, $k_{3}, k_{4}, k_{5}$ and $k_{6}$ were fixed at their initial values.

The preliminary 100 sets of parameter estimates obtained via Bayesian and LO estimation were then used to design 100 sets of sequential A-optimal experiments using the Bayesian and LO approaches. Next, the 100 sets of designed experiments were used to generate 100 new datasets (using the true parameter values and random measurement errors). Details concerning how many and which parameters tended to be estimated after each stage of sequential experimentation are provided in the Supplementary Information. 
Figure 3 provides boxplots for 100 values of the scaled sum of squared deviations between the estimated and true parameter values:

$$
S S D_{\theta}=\left(\widehat{\boldsymbol{\theta}}-\boldsymbol{\theta}^{\text {true }}\right)^{T} \boldsymbol{\Sigma}_{\mathbf{0}}^{-\mathbf{1}}\left(\widehat{\boldsymbol{\theta}}-\boldsymbol{\theta}^{\text {true }}\right)
$$

for all four approaches when selecting three new A-optimal experiments, one at a time. For fair comparison between all four approaches when using equation (19), we do not entirely neglect the left-out parameters, but instead fix them at their initial guesses, which are based on engineering judgment. In this way, all the model parameters (both the estimated ones and those that are fixed at their initial values) are considered for computation of $S S D_{\theta}$. The Figure 3 shows the box plots for $S S D_{\theta}$ for all for approaches. In Figure 3, the titles $1^{\text {st }}, 2^{\text {nd }}$ and $3^{\text {rd }}$ round indicate the stage of experimentation. One new experiment is performed during each round. The Bayes-LO approach is the superior approach on average, resulting in the smallest mean and median for $S S D_{\theta}$ after each round of experimentation. The results in Figure 3 indicate that designing experiments using the proposed modified Bayesian approach (i.e., with equation (16) as the objective function) is superior to designing experiments using the LO approach (i.e., the BayesBayes results are better than LO-Bayes, and the Bayes-LO results are better than LO-LO). In addition, parameter estimation using the LO approach is superior to the Bayesian approach (i.e., Bayes-LO is better than Bayes-Bayes, and LO-LO is better than LO-Bayes).

Figure 4 shows boxplots for 100 values of $S S D_{\theta}$ when designing three new A-optimal experiments all at once for Case I. Bayes-LO is the superior approach and LO-Bayes is the worst approach. Comparing these results with the results in Figure 3, it can be concluded that designing experiments one-at-a-time resulted in better final parameter values than designing all three new experiments at once. It is not 
surprising that designing experiments one-at-a-time resulted in better final parameter estimates than designing all three experiments at once. Note that, when designing experiments one-at-a-time, the parameter values were updated after each iteration. However, scaling factors for the initial parameter uncertainties (which reflect prior parameter knowledge) were not changed because information obtained during all previous experimental runs is contained in $\mathbf{Z}_{\text {old. }}$. It is important that this information should not be double-counted.

Figure 3. Boxplots for 100 values of $S S D_{\theta}$ for Case I, when designing three sequential A-optimal experiments one at a time using LO-LO, Bayes-LO, LO-Bayes, and Bayes-Bayes approaches

Figure 4. Boxplots for 100 values of $S S D_{\theta}$ for Case I, when designing three sequential A-optimal experiment all at once using Bayes-Bayes, LO-LO, Bayes-LO, and LO-Bayes approaches

Computation times for both Bayesian and LO approaches to MBDOE were compared using MC simulations for the Bayes-Bayes and LO-Bayes approaches. These two approaches use the same approach for parameter estimation, but different methods for MBDOE, making it possible to isolate the effects of Dayesian and LO approaches for MBDoE. All computations were performed in a core i5 laptop with 8 GB RAM which resulted in the average computations time of $51.2 \mathrm{~s}$ for each Bayes-Bayes run and 89.9 s for each Bayes-Bayes run. This result indicates that the proposed simple Bayesian experimental design was faster on average compared to LO experimental design. Although this difference is relatively small 
for the current case study, we anticipate that larger differences could occur for larger models with more parameters and decision variables.

\section{Case II: Results when moderately-informative initial guesses are available}

Figure 5 compares boxplots for the 100 values of $S S D_{\theta}$ for four approaches when designing three A-optimal experiments one at a time using the prior parameter guesses in Case II. Similar patterns are observed compared to Case I: as more experiments are designed and more data become available, the mean and median values of boxplots for $S S D_{\theta}$ becomes smaller. However, since the parameter initial guesses are not as good as in Case I, the parameter estimates in Case II are less accurate than in Case I. As in Case I, the Bayes-LO approach provides the best parameter values on average. A key difference between the results in Case I and Case II is that the LO approach tended to estimate more parameters in Case II, due to higher initial parameter uncertainties. Details concerning the frequency with which different parameters were estimated are provided in the Supplementary Information.

\section{Case III: Results when misinformed initial guesses are used}

Figure 6 shows boxplots for 100 values of $S S D_{\theta}$ obtained for Case III, with the misinformed parameter initial guesses described in the third row of Table 7. As expected, because parameter initial guesses were worse than in Case I and II, the mean and median for $S S D_{\theta}$ are larger than Case I and II for all four approaches. The Bayes-LO approach to designing experiments and estimating parameters was the best approach, even though the modeler believed he or she had better prior knowledge about the parameters than was justifiable. These results suggest that the Bayes-LO approach is somewhat robust to specification of misinformed prior information. Note that the settings in Case II and Case III were also 
used to design three experiments all-at-once instead of one at a time. As in Case I, the Bayes-LO approach was the best and the parameter estimations resulting from the three-at-once experiments were not as good as those obtained using the one-at-a-time approach. Details are provided in the Supplementary Information.

Cases I, II and III were also repeated using the extended 14 parameter model that arises (due to activation energies and reaction enthalpy) when the temperature is included as an additional decision variable. The same trends were observed as for the 7 parameters model, indicating that the Bayes-LO is the best of the four approaches studied. Details are provided in the Supplementary Information.

Figure 5. Boxplots for 100 values of $S S D_{\theta}$ for Case II, when designing three sequential A-optimal experiment one at a time using LO-LO, Bayes-LO, LO-Bayes, and Bayes-Bayes approaches

\section{CONCLUSIONS}

In this study, we developed a new Tikhonov based approach for MBDoE to address the problem of ill-conditioned/singular FIM. The Tikhonov weightings are specified by using Bayesian arguments based on prior knowledge of the modeler about plausible values of the parameters. This approach has never been used before and results in a simplified Bayesian MBDoE objective function that can be used during sequential model-based design of experiments (MBDoE) when the FIM is noninvertible. The results for the proposed Bayesian approach were compared with a leave-out (LO) approach developed in previous studies. In addition, the effectiveness of Bayesian and LO approaches for parameter estimation were also compared, so that four different approaches were investigated (i.e., Bayes-Bayes, LO-LO, 
Bayes-LO, and LO-Bayes) were investigated. These approaches were tested using simulated data generated from a 7-parameter isothermal pharmaceutical production model and a corresponding 14parameter non-isothermal model. Three different cases were considered wherein the modeler specified different prior information about the parameters. The results indicate that the Bayes-LO approach (i.e., a Bayesian approach for MBDoE combined with a LO approach for parameter estimations) is superior to the three other approaches. The proposed Bayesian approach for designing experiments consistently provided superior experiments for use in parameter estimation compared with the LO approach. However, after new experimental data had been obtained, the LO approach for parameter estimation consistently provided parameter values that were closer, on average, to their true values than parameter estimates obtained from Bayesian estimation. Promising simulation results obtained using misspecified prior parameter knowledge indicated that the Bayes-LO approach was somewhat robust to misinformation for the current case study. The reasons that the Bayes-LO approach is superior to the other approaches is unclear. Experience with additional case studies will be beneficial for investigating whether this finding is typical. 
Figure 6. Boxplots for 100 values of $S S D_{\theta}$ for Case III, when designing three sequential A-optimal experiment one at a time using Bayes-Bayes, LO-LO, Bayes-LO, and LO-Bayes approaches

\section{ACKNOWLEDGMENT}

Authors acknowledge financial support from Eli Lilly and Company and technical advice from Dr.

Salvador Garcia-Munoz and Dr. Brandon Jacob Reizman. An Ontario Trillium Scholarship for A. Shahmohammadi from government from the province of Ontario is also gratefully acknowledged.

\section{REFERENCES}

1. Okino MS, Mavrovouniotis ML. Simplification of mathematical models of chemical reaction systems. Chem Rev. Published online 1998. doi:10.1021/cr950223l

2. Dobre TG, Sanchez Marcano JG. Chemical Engineering: Modelling, Simulation and Similitude.; 2007. doi:10.1002/9783527611096

3. Birtwistle MR, Mager DE, Gallo JM. Mechanistic Vs. empirical network models of drug action. CPT Pharmacometrics Syst Pharmacol. Published online 2013. doi:10.1038/psp.2013.51

4. Gernaey K V., Lantz AE, Tufvesson P, Woodley JM, Sin G. Application of mechanistic models to fermentation and biocatalysis for next-generation processes. Trends Biotechnol. Published online 2010. doi:10.1016/j.tibtech.2010.03.006

5. Boukouvala F, Muzzio FJ, Ierapetritou MG. Dynamic data-driven modeling of pharmaceutical processes. Ind Eng Chem Res. Published online 2011. doi:10.1021/ie102305a

6. Domagalski NR, Mack BC, Tabora JE. Analysis of Design of Experiments with Dynamic Responses. Org Process Res Dev. Published online 2015. doi:10.1021/acs.oprd.5b00143 
7. Feng AL, Boraey MA, Gwin MA, Finlay PR, Kuehl PJ, Vehring R. Mechanistic models facilitate efficient development of leucine containing microparticles for pulmonary drug delivery. Int $J$ Pharm. Published online 2011. doi:10.1016/j.ijpharm.2011.02.049

8. Kourti T, Lepore J, Liesum L, et al. Scientific and Regulatory Considerations for Implementing Mathematical Models in the Quality by Design (QbD) Framework. Pharm Eng. Published online 2015.

9. Karlsson S, Rasmuson A, Van WaChem B, Björn IN. CFD modeling of the wurster bed coater. AIChE J. Published online 2009. doi:10.1002/aic.11847

10. Maria G. A review of algorithms and trends in kinetic model identification for chemical and biochemical systems. Chem Biochem Eng Q. 2004;18(3):195-222.

11. Atkinson AC. Developments in the Design of Experiments, Correspondent Paper. Int Stat Rev / Rev Int Stat. 1982;50(2):161-177. doi:10.2307/1402599

12. Liu S, Neudecker H. A V-optimal design for Scheffé's polynomial model. Stat Probab Lett. 1995;23(3):253-258. doi:10.1016/0167-7152(94)00122-O

13. Chaloner K, Verdinelli I. Bayesian experimental design: A review. Stat Sci. 1995;10(3):273-304. doi:10.1214/ss/1177009939

14. Galvanin F, Barolo M, Bezzo F. Online model-based redesign of experiments for parameter estimation in dynamic systems. Ind Eng Chem Res. 2009;48(9):4415-4427. doi:10.1021/ie8018356

15. John RCS, Draper NR. D-Optimality for regression designs: A review. Technometrics. 
1975;17(1):15-23. doi:10.1080/00401706.1975.10489266

16. Krafft O, Schaefer M. D-optimal designs for a multivariate regression model. J Multivar Anal. 1992;42(1):130-140. doi:10.1016/0047-259X(92)90083-R

17. Franceschini G, Macchietto S. Model-based design of experiments for parameter precision: State of the art. Chem Eng Sci. Published online 2008. doi:10.1016/j.ces.2007.11.034

18. Box GEP, Lucas HL. Design of Experiments in Non-Linear Situations. Vol 46. [Oxford University Press, Biometrika Trust]; 1959.

19. Goos P, Syafitri U. V-optimal mixture designs for the qth degree model. Chemom Intell Lab Syst. 2014;136:173-178. doi:10.1016/j.chemolab.2014.04.019

20. Wong WK. Comparing robust properties of A, D, E and G-optimal designs. Comput Stat Data Anal. Published online 1994. doi:10.1016/0167-9473(94)90161-9

21. Atkinson AC, Fedorov V V. The design of experiments for discriminating between two rival models. Biometrika. Published online 1975. doi:10.1093/biomet/62.1.57

22. Banga JR, Balsa-Canto E. Parameter estimation and optimal experimental design. Essays Biochem. Published online 2008. doi:10.1042/bse0450195

Stigler SM. Optimal experimental design for polynomial regression. J Am Stat Assoc. 1971;66(334):311-318.

24. Shahmohammadi A. Model-based optimal design of experiments with noninvertible fisher information matrix. Published online 2019.

25. Shahmohammadi A, McAuley KB. Sequential Model-Based A- and V-Optimal Design of 
Experiments for Building Fundamental Models of Pharmaceutical Production Processes. Comput Chem Eng. Published online 2019.

26. López DC, Barz T, Peñuela M, Villegas A, Ochoa S, Wozny G. Model-based identifiable parameter determination applied to a simultaneous saccharification and fermentation process model for bio-ethanol production. Biotechnol Prog. 2013;29(4):1064-1082.

doi:10.1002/btpr.1753

27. Walter E, Pronzato L. Qualitative and quantitative experiment design for phenomenological models-A survey. Automatica. 1990;26(2):195-213. doi:10.1016/0005-1098(90)90116-Y

28. Pinto JC, Lobão MW, Monteiro JL. Sequential experimental design for parameter estimation: a different approach. Chem Eng Sci. 1990;45(4):883-892. doi:http://dx.doi.org/10.1016/00092509(90)85010-B

29. Ford I, Silvey SD. A sequentially constructed design for estimating a nonlinear parametric function. Biometrika. 1980;67(2):381-388. doi:10.1093/biomet/67.2.381

30. Issanchou S, Cognet P, Cabassud M. Sequential experimental design strategy for rapid kinetic modeling of chemical synthesis. AIChE J. 2005;51(6):1773-1781. doi:10.1002/aic.10439 Bauer I, Bock HG, Körkel S, Schlöder JP. Numerical methods for optimum experimental design in DAE systems. J Comput Appl Math. 2000;120(1):1-25. doi:http://dx.doi.org/10.1016/S03770427(00)00300-9

32. Jacquez JA, Greif P. Numerical parameter identifiability and estimability: Integrating identifiability, estimability, and optimal sampling design. Math Biosci. Published online 1985. 
doi:10.1016/0025-5564(85)90098-7

33. Mclean KAP, Mcauley KB. Mathematical modelling of chemical processes-obtaining the best model predictions and parameter estimates using identifiability and estimability procedures. Can J Chem Eng. 2012;90(2):351-366. doi:10.1002/cjce.20660

34. VAJDA S, RABITZ H, WALTER E, LECOURTIER Y. QUALITATIVE AND QUANTITATIVE IDENTIFIABILITY ANALYSIS OF NONLINEAR CHEMICAL KINETIC MODELS. Chem Eng Commun. 1989;83(1):191-219. doi:10.1080/00986448908940662

35. Littlejohns J V, McAuley KB, Daugulis AJ. Model for a solid-liquid stirred tank two-phase partitioning bioscrubber for the treatment of BTEX. J Hazard Mater. 2010;175(1):872-882. doi:http://dx.doi.org/10.1016/j.jhazmat.2009.10.091

36. Ben-Zvi A, McAuley K, McLellan J. Identifiability study of a liquid-liquid phase-transfer catalyzed reaction system. AIChE J. 2004;50(10):2493-2501. doi:10.1002/aic.10202

37. Zhao YR, Arriola DJ, Puskas JE, McAuley KB. Applying multidimensional method of moments for modeling and estimating parameters for arborescent polyisobutylene production in batch reactor. Macromol Theory Simulations. 2017;26(1). doi:10.1002/mats.201600004

Issanchou S, Cognet P, Cabassud M. Precise parameter estimation for chemical batch reactions in heterogeneous medium. Chem Eng Sci. 2003;58(9):1805-1813. doi:10.1016/S00092509(03)00004-6

39. Cho KH, Shin SY, Kolch W, Wolkenhauer O. Experimental Design in Systems Biology, Based on Parameter Sensitivity Analysis Using a Monte Carlo Method: A Case Study for the TNF - 
Mediated NF- B Signal Transduction Pathway. Simulation. Published online 2003. doi:10.1177/0037549703040943

40. López C. DC, Barz T, Körkel S, Wozny G. Nonlinear ill-posed problem analysis in model-based parameter estimation and experimental design. Comput Chem Eng. 2015;77:24-42. doi:10.1016/j.compchemeng.2015.03.002

41. Thompson DE, McAuley KB, McLellan PJ. Design of optimal sequential experiments to improve model predictions from a polyethylene molecular weight distribution model. Macromol React Eng. 2010;4(1):73-85. doi:10.1002/mren.200900033

42. Greville TNE. The pseudoinverse of a rectangular or singular matrix and its application to the solution of systems of linear equations. SIAM Rev. 1959;1(1):38-43. doi:10.1137/1001003

43. Tibshirani R. Regression shrinkage and selection via the lasso: a retrospective. J R Stat Soc Ser B (Statistical Methodol. 2011;73(3):273-282. doi:10.1111/j.1467-9868.2011.00771.x

44. Johansen TA. On Tikhonov regularization, bias and variance in nonlinear system identification. Automatica. 1997;33(3):441-446. doi:http://dx.doi.org/10.1016/S0005-1098(96)00168-9

45. Tikhonov AN, Goncharsky A V., Stepanov V V., Yagola AG. Numerical methods for the solution of ill-posed problems. Math Comput. 1978;32(144):1320. doi:10.2307/2006360

46. Hoerl AE, Kennard RW. Ridge Regression: Biased estimation for nonorthogonal problems. Technometrics. 1970;12(1):55. doi:10.2307/1267351

47. Durán MA, White BS. Bayesian estimation applied to effective heat transfer coefficients in a packed bed. Chem Eng Sci. 1995;50(3):495-510. doi:http://dx.doi.org/10.1016/0009- 
2509(94)00260-X

48. Ruggoo A, Vandebroek M. Bayesian sequential script dsign optimal model-robust designs. Comput Stat Data Anal. 2004;47(4):655-673. doi:10.1016/j.csda.2003.09.014

49. Kravaris C, Hahn J, Chu Y. Advances and selected recent developments in state and parameter estimation. Comput Chem Eng. 2013;51:111-123. doi:10.1016/j.compchemeng.2012.06.001

50. Barz T, López Cárdenas DC, Arellano-Garcia H, Wozny G. Experimental evaluation of an approach to online redesign of experiments for parameter determination. AIChE J. Published online 2013. doi:10.1002/aic.13957

51. Yao KZ, Shaw BM, Kou B, McAuley KB, Bacon DW. Modeling Ethylene/Butene copolymerization with multi-site catalysts: parameter estimability and experimental design. Polym React Eng. 2003;11(3):563-588. doi:10.1081/PRE-120024426

52. Shahmohammadi A, McAuley KB. Sequential Model-Based A-Optimal Design of Experiments When the Fisher Information Matrix Is Noninvertible. Ind Eng Chem Res. 2019;58(3):1244-1261. doi:10.1021/acs.iecr.8b03047

53. Dubé MA, Penlidis A, Reilly PM. A systematic approach to the study of multicomponent polymerization kinetics: The butyl acrylate/methyl methacrylate/vinyl acetate example. IV. Optimal Bayesian design of emulsion terpolymerization experiments in a pilot plant reactor. $J$ Polym Sci Part A Polym Chem. Published online 1996. doi:10.1002/(SICI)10990518(19960415)34:5<811::AID-POLA11>3.3.CO;2-3

54. Hsu SH, Stamatis SD, Caruthers JM, et al. Bayesian framework for building kinetic models of 
catalytic systems. Ind Eng Chem Res. Published online 2009. doi:10.1021/ie801651y

55. Han C, Chaloner K. Bayesian Experimental Design for Nonlinear Mixed-Effects Models with Application to HIV Dynamics. Biometrics. Published online 2004. doi:10.1111/j.0006341X.2004.00148.X

56. Van Den Berg J, Curtis A, Trampert J. Optimal nonlinear Bayesian experimental design: An application to amplitude versus offset experiments. Geophys J Int. Published online 2003. doi:10.1046/j.1365-246X.2003.02048.X

57. Ryan EG, Drovandi CC, Pettitt AN. Simulation-based fully Bayesian experimental design for mixed effects models. Comput Stat Data Anal. Published online 2015.

doi:10.1016/j.csda.2015.06.007

58. Jaakkola TS, Jordan MI. Bayesian parameter estimation via variational methods. Stat Comput. Published online 2000. doi:10.1023/A:1008932416310

59. Lehninger. Principles of Biochemistry, 6th Ed.; 2009. doi:10.1017/CBO9781107415324.004

60. Petersen B, Gernaey K, Vanrolleghem PA. Practical identifiability of model parameters by combined respirometric-titrimetric measurements. In: Water Science and Technology. ; 2001.

Thompson DE, McAuley KB, McLellan PJ. Parameter estimation in a simplified MWD model for HDPE produced by a ziegler-natta catalyst. Macromol React Eng. 2009;3(4):160-177. doi:10.1002/mren.200800052

62. Lin W, Biegler LT, Jacobson AM. Modeling and optimization of a seeded suspension polymerization process. Chem Eng Sci. 2010;65(15):4350-4362. doi:10.1016/j.ces.2010.03.052 
63. Wu S, McLean K a. P, Harris TJ, McAuley KB. Selection of optimal parameter set using estimability analysis and MSE-based model-selection criterion. Int J Adv Mechatron Syst. 2011;3(3):188. doi:10.1504/IJAMECHS.2011.042615

64. Wu S, Mcauley KB, Harris TJ. Selection of simplified models: II. Development of a model selection criterion based on mean squared error. Can J Chem Eng. 2011;89(2):325-336. doi:10.1002/cjce.20479

65. Chu Y, Hahn J. Parameter set selection via clustering of parameters into pairwise indistinguishable groups of parameters. Ind Eng Chem Res. Published online 2009. doi:10.1021/ie800432s

66. LORD FM. MAXIMUM LIKELIHOOD AND BAYESIAN PARAMETER ESTIMATION IN ITEM RESPONSE THEORY. J Educ Meas. Published online 1986. doi:10.1111/j.17453984.1986.tb00241.x

67. Bates DM, Watts DG. Review of Linear Regression. Nonlinear Regres Anal Its Appl. Published online 1988:1-31. doi:10.1002/9780470316757.ch1

68. Fabian B, Bernd H. Uncertainty estimation for linearised inverse problems comparing Bayesian inference and a pseudoinverse approach for acoustic transmission measurements. tm - Tech Mess. 2017;84:217. doi:10.1515/teme-2016-0022

69. Englezos P, Kalogerakis N. Applied Parameter Estimation for Chemical Engineers. Vol 53.; 1989. doi:10.1017/CBO9781107415324.004

70. Bard Y. Nonlinear Parameter Estimation. Oper Res Q 19701977. 1974;49(3):341. 
71. Galvanin F, Macchietto S, Bezzo F. Model-based design of parallel experiments. Ind Eng Chem Res. Published online 2007. doi:10.1021/ie0611406

72. Ryan KJ. Estimating Expected Information Gains for Experimental Designs with Application to the Random Fatigue-Limit Model. J Comput Graph Stat. Published online 2003. doi:10.1198/1061860032012

73. Cox DR, Reid N. The Theory of the Design of Experiments. Chapman and Hall/CRC; 2000.

74. DuMouchel W, Jones B. A simple bayesian modification of d-optimal designs to reduce dependence on an assumed model. Technometrics. Published online 1994. doi:10.1080/00401706.1994.10485399

75. Vivaldo-Lima E, Penlidis A, Wood PE, Hamielec AE. Determination of the relative importance of process factors on particle size distribution in suspension polymerization using a bayesian experimental design technique. J Appl Polym Sci. Published online 2006. doi:10.1002/app.24889

76. Lindley D V. On a Measure of the Information Provided by an Experiment. Ann Math Stat. 1956;27(4):986-1005. doi:10.1214/aoms/1177728069

77. Papadimitriou C, Argyris C. Bayesian optimal experimental design for parameter estimation and response predictions in complex dynamical systems. In: Procedia Engineering. ; 2017. doi:10.1016/j.proeng.2017.09.205

78. Alexanderian A, Gloor PJ, Ghattas O. On Bayesian A- and D-optimal experimental designs in infinite dimensions. Bayesian Anal. Published online 2016. doi:10.1214/15-BA969

79. Papadimitriou C, Beck JL, Au SK. Entropy-based optimal sensor location for structural model 
updating. JVC/Journal Vib Control. Published online 2000. doi:10.1177/107754630000600508

80. Sacks J, Welch WJ, Mitchell TJ, Wynn HP. Design and Analysis of Computer Experiments. Stat Sci. 1989;4(4):409-423. 


\section{LIST OF FIGURE CAPTIONS}

Figure 1. Reaction scheme for Michaelis-Menten case study

Figure 2. Simulated data (symbols) and noise-free concentration profiles (curves) obtained at 40 ${ }^{\circ} \mathrm{C}$ using experimental settings in Table 3 , kinetic coefficients in Table 4 and measurement errors in Table 5: a) SM1; b) D; c) SM2; d) P

Figure 3. Boxplots for 100 values of $S S D_{\theta}$ for Case I, when designing three sequential A-optimal experiments one at a time using LO-LO, Bayes-LO, LO-Bayes, and Bayes-Bayes approaches

Figure 4. Boxplots for 100 values of $S S D_{\theta}$ for Case I, when designing three sequential A-optimal experiment all at once using Bayes-Bayes, LO-LO, Bayes-LO, and LO-Bayes approaches

Figure 5. Boxplots for 100 values of $S S D_{\theta}$ for Case II, when designing three sequential A-optimal experiment one at a time using LO-LO, Bayes-LO, LO-Bayes, and Bayes-Bayes approaches

Figure 6. Boxplots for 100 values of $S S D_{\theta}$ for Case III, when designing three sequential A-optimal experiment one at a time using Bayes-Bayes, LO-LO, Bayes-LO, and LO-Bayes approaches 


\section{LIST OF TABLE CAPTIONS}

Table 1. Optimality criteria for model-based design of experiments

Table 2. Orthogonalization algorithm

Table 3. Initial conditions for center-point batch reactor operation at $\mathrm{T}=40^{\circ} \mathrm{C}$

Table 4. True values of the kinetic coefficients and equilibrium constant

Table 5. Measurement variances used for generating simulated data

Table 6. Lower and upper bounds for the decision variables

Table 7. Selection of parameter initial guesses from normal distributions 


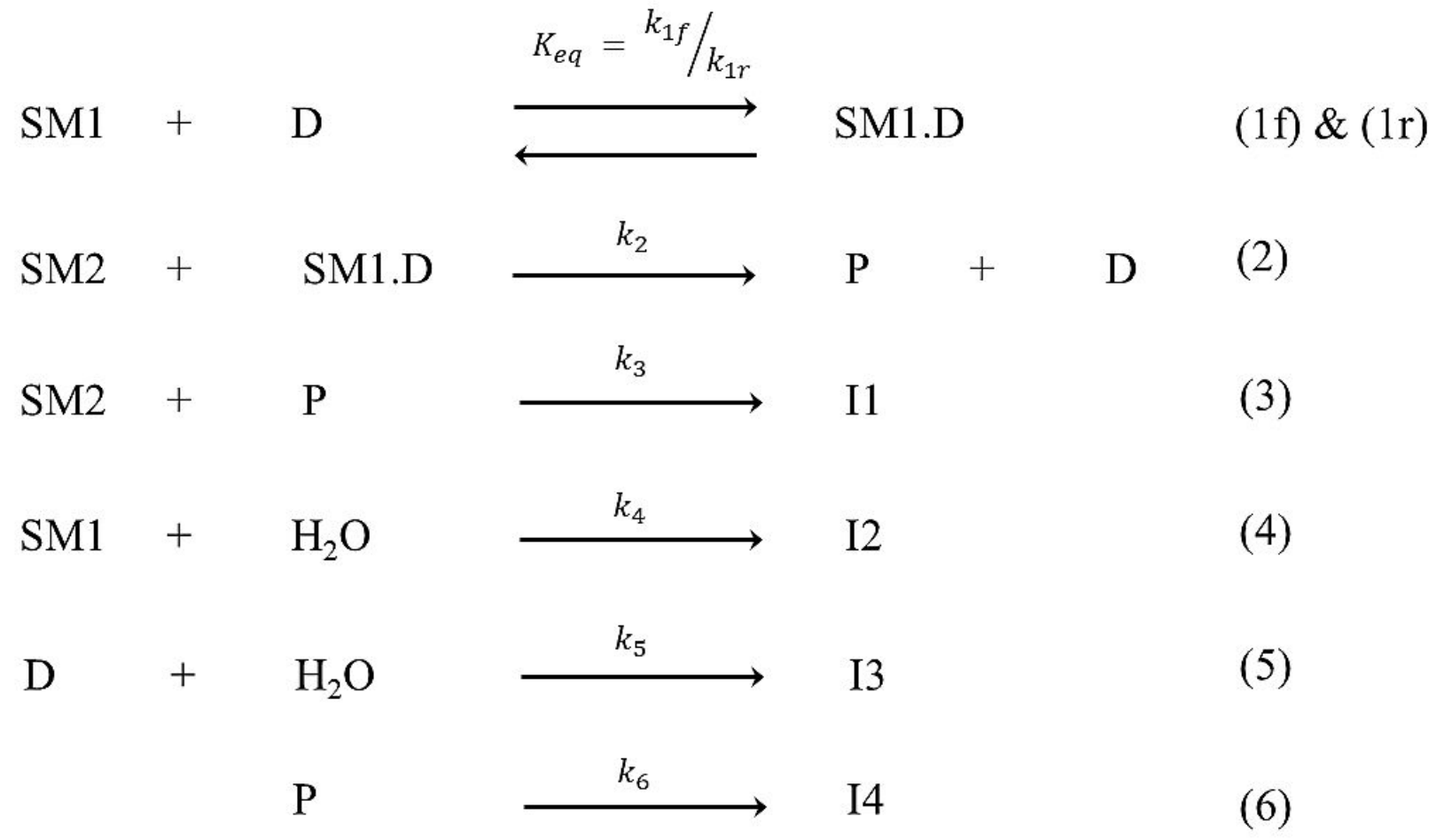

Figure 1. Reaction scheme for Michaelis-Menten case study ${ }^{6}$ 

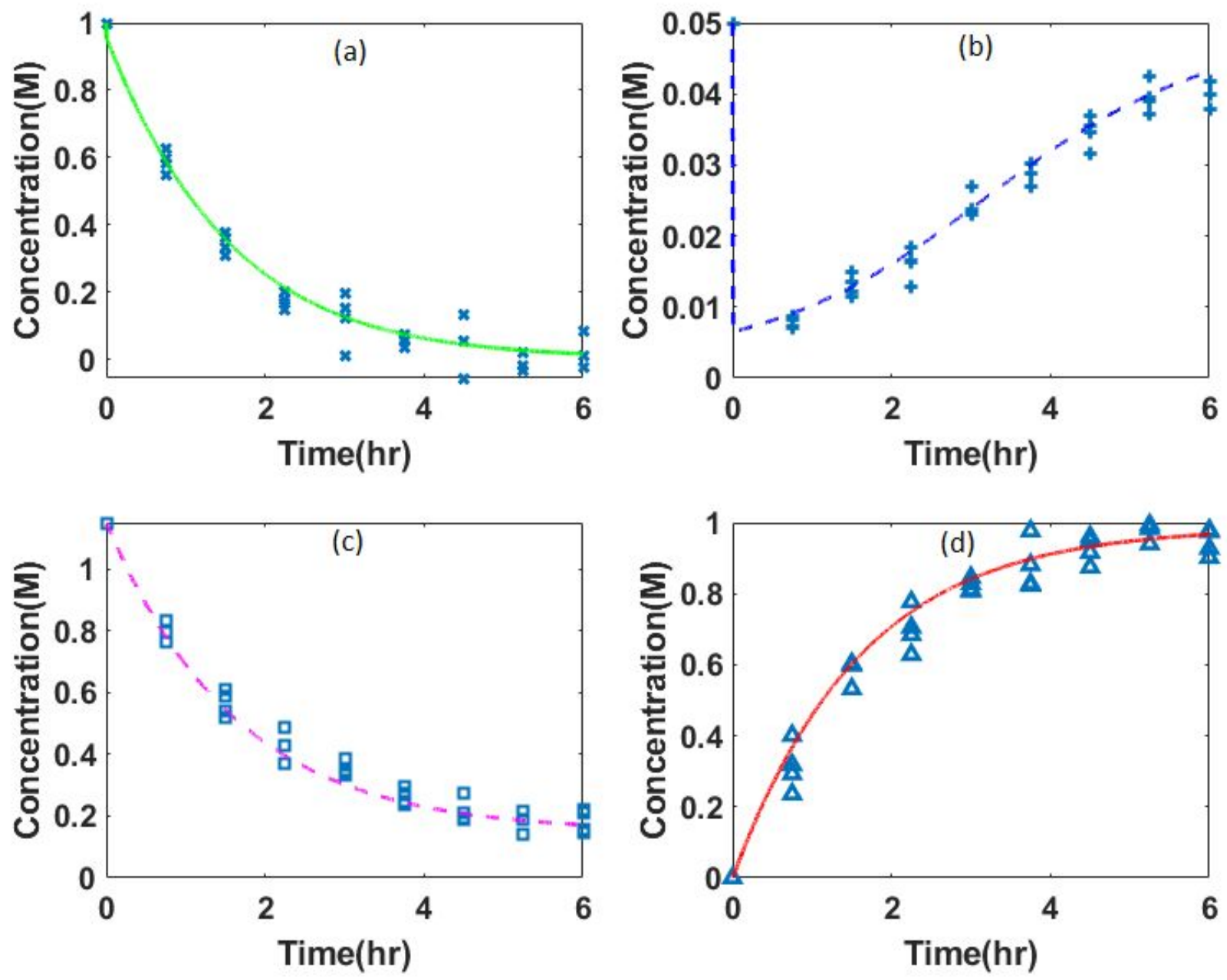

Figure 2. Simulated data (symbols) and noise-free concentration profiles (curves) obtained at 40 ${ }^{\circ} \mathrm{C}$ using experimental settings in Table 3 , kinetic coefficients in Table 4 and measurement errors in Table 5: a) SM1; b) D; c) SM2; d) P. 

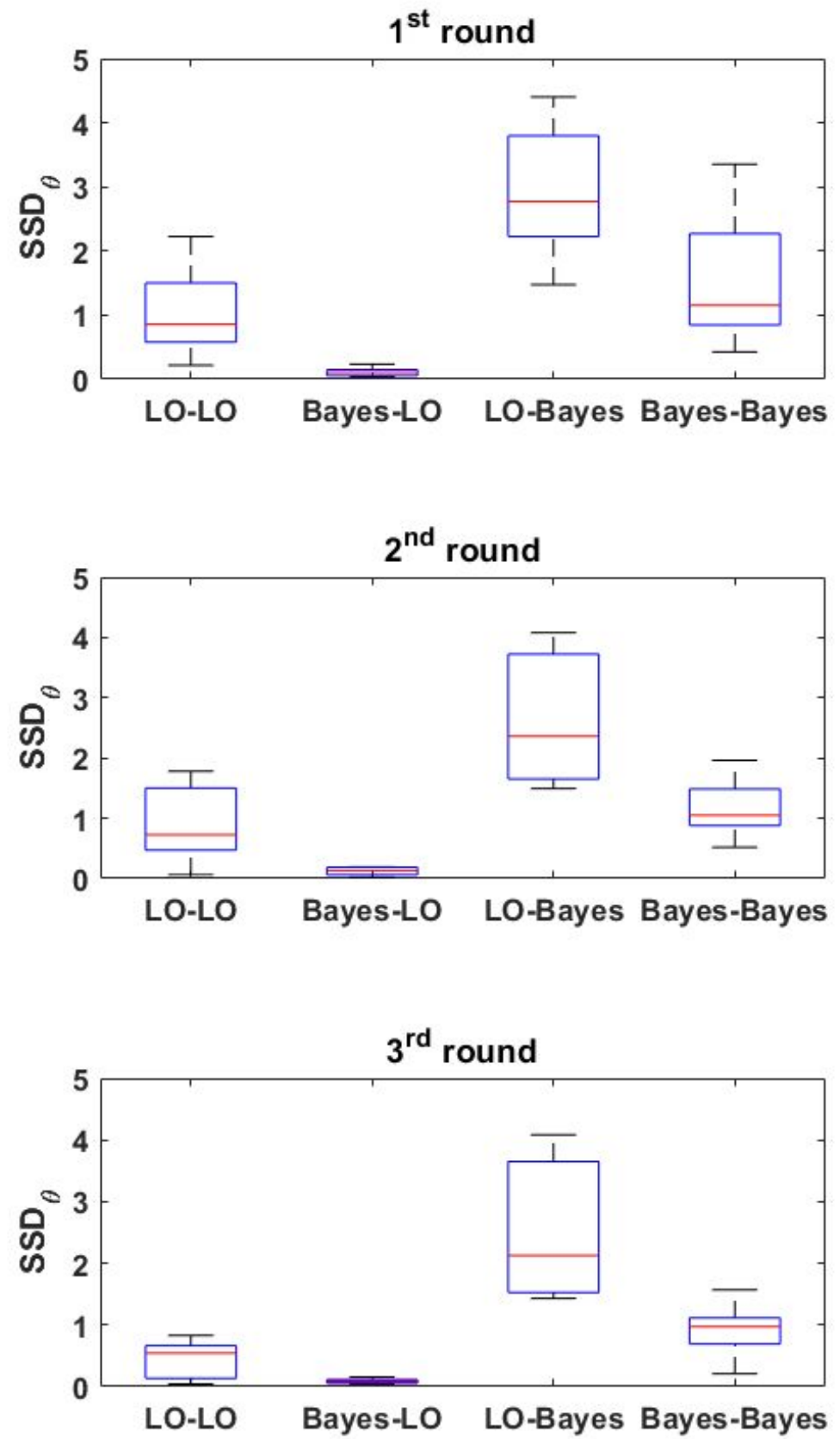

Figure 3. Boxplots for 100 values of $S S D_{\theta}$ for Case I, when designing three sequential A-optimal experiments one at a time using LO-LO, Bayes-LO, LO-Bayes, and Bayes-Bayes approaches 


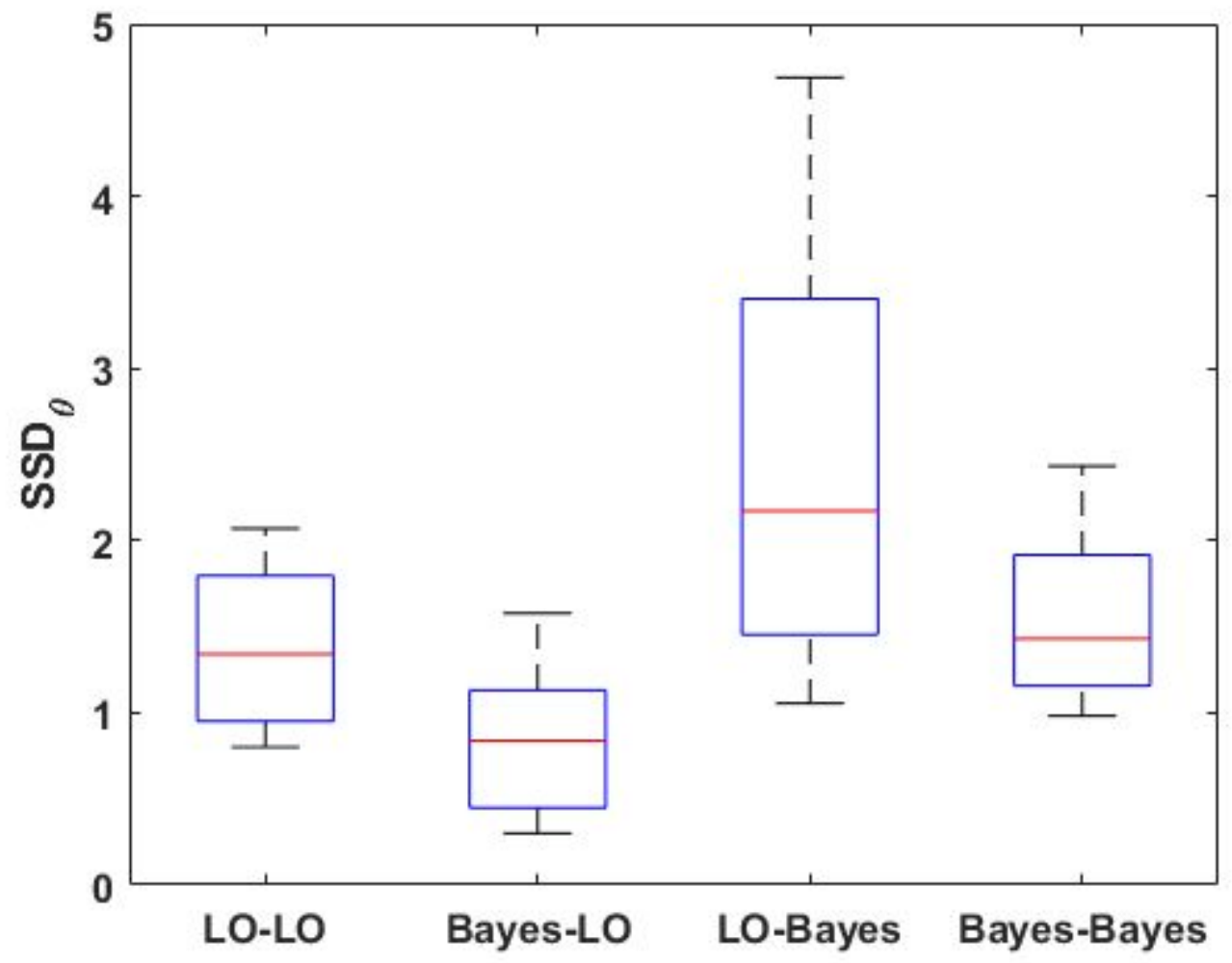

Figure 4. Boxplots for 100 values of $S S D_{\theta}$ for Case I, when designing three sequential A-optimal experiment all at once using Bayes-Bayes, LO-LO, Bayes-LO, and LO-Bayes approaches 

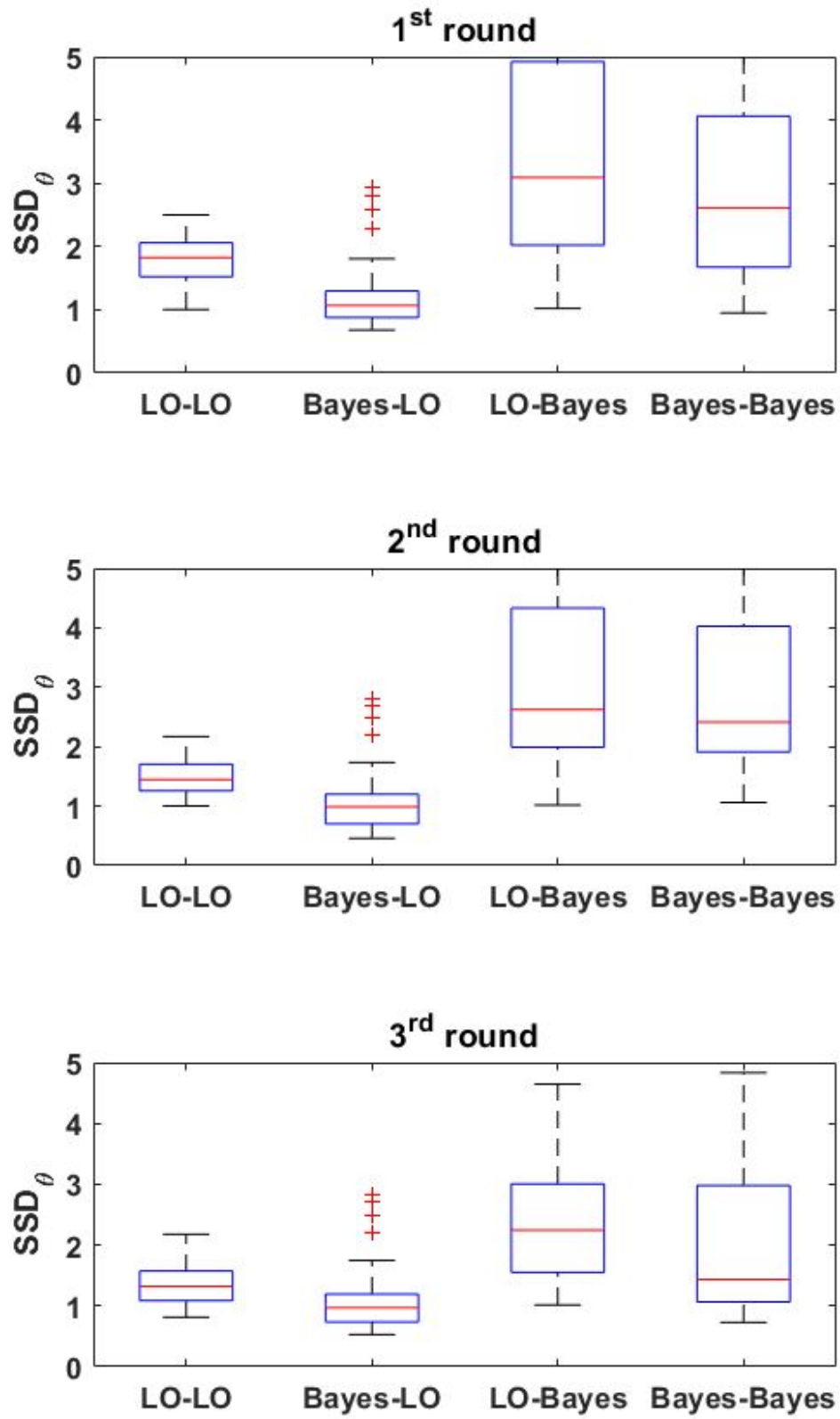

Figure 5. Boxplots for 100 values of $S S D_{\theta}$ for Case II, when designing three sequential A-optimal experiment one at a time using LO-LO, Bayes-LO, LO-Bayes, and Bayes-Bayes approaches 

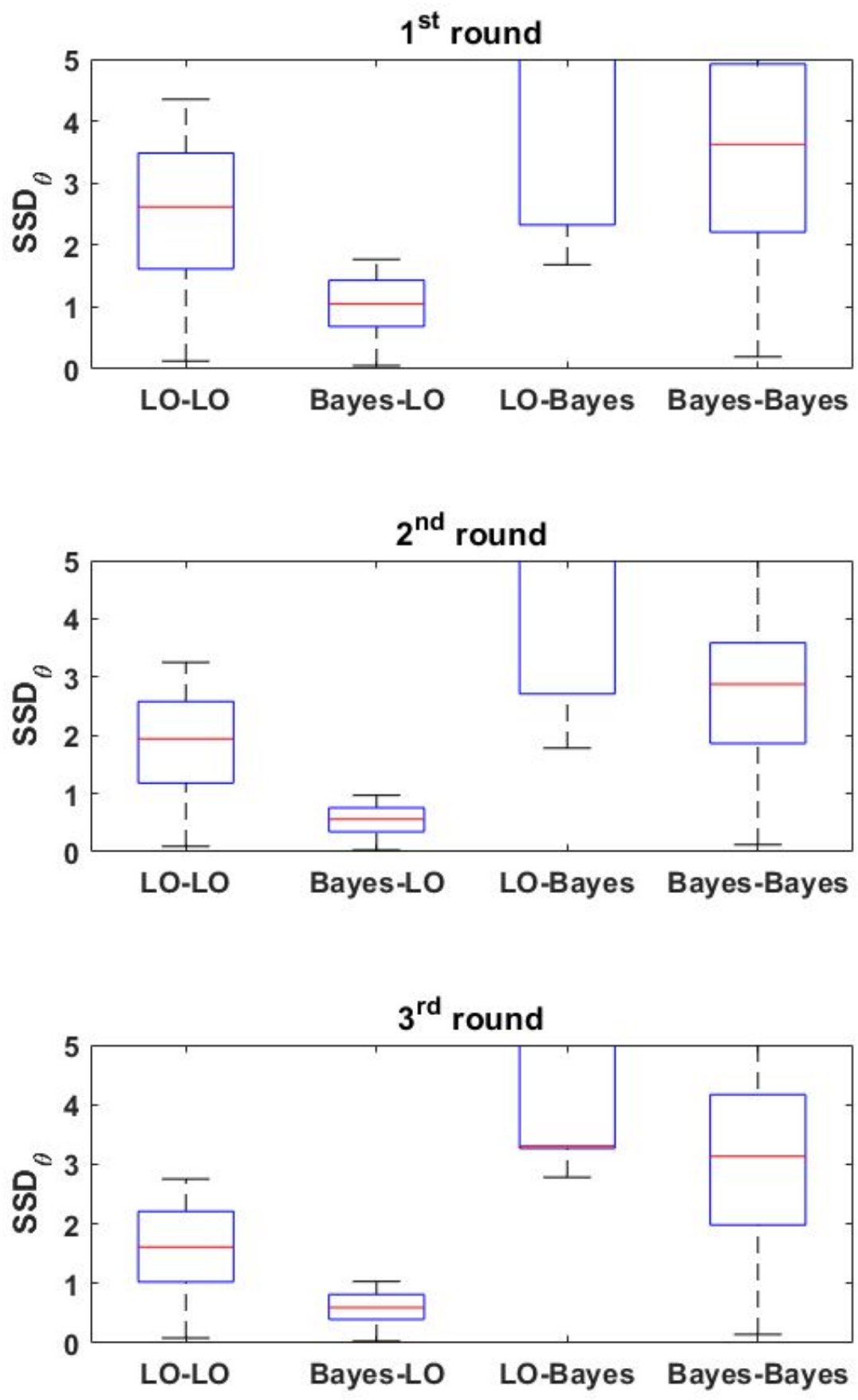

Figure 6. Boxplots for 100 values of $S S D_{\theta}$ for Case III, when designing three sequential A-optimal experiment one at a time using Bayes-Bayes, LO-LO, Bayes-LO, and LO-Bayes approaches 
Table 1. Optimality criteria for model-based design of experiments

\begin{tabular}{|c|c|c|}
\hline Optimality Criterion & Description & $\begin{array}{l}\text { Eq. } \\
\#\end{array}$ \\
\hline$J_{A}=\operatorname{trace}\left((\mathbf{F I M})^{-1}\right)$ & A-optimal design minimizes total parameter variance. & $(1.1)$ \\
\hline$J_{D}=\operatorname{det}\left((\mathbf{F I M})^{-1}\right)$ & $\begin{array}{l}\text { D-optimal design minimizes the volume of the joint } \\
\text { confidence interval for the parameters. }\end{array}$ & $(1.2)$ \\
\hline$J_{E}=\lambda_{\max }\left(\mathbf{F I M}^{-1}\right)$ & $\begin{array}{l}\text { E-optimal design minimizes the largest eigenvalue of the } \\
\text { FIM, thereby minimizing the uncertainties in the worst- } \\
\text { case direction in the parameter space. }\end{array}$ & $(1.3)$ \\
\hline$J_{G}=\max \left(\operatorname{diag}\left(\mathbf{W}(\mathbf{F I} \mathbf{M})^{-1} \mathbf{W}^{\mathbf{T}}\right)\right)$ & $\begin{array}{l}\text { Multi-response G-optimal design minimizes the } \\
\text { maximum variance of model predictions at user-specified } \\
\text { operating conditions of interest, specified using a matrix } \mathbf{W} \text {. } \\
\text { This is equivalent to minimizing the largest value of the } \\
\text { diagonal elements of } \mathbf{W}(\mathbf{F I M})^{-1} \mathbf{W}^{\mathbf{T}} \text {. }\end{array}$ & $(1.4)$ \\
\hline$J_{V}=\operatorname{trace}\left(\mathbf{W}(\mathbf{F I M})^{-1} \mathbf{W}^{\mathbf{T}}\right)$ & $\begin{array}{l}\text { Multi-response V-optimal design minimizes the total } \\
\text { variance of model predictions at user-specified operating } \\
\text { conditions of interest, which are specified using matrix } \mathbf{W} \text {. } \\
\text { This is equivalent to minimizing the trace of } \mathbf{W}(\mathbf{F I M})^{-1} \\
\mathbf{W}^{\mathbf{T}} \text {. }\end{array}$ & $(1.5)$ \\
\hline
\end{tabular}

This article is protected by copyright. All rights reserved. 
Table 2. Orthogonalization algorithm ${ }^{51,61}$

1. Compute the magnitude (i.e., the Euclidean norm) of each column in the $\mathbf{Z}$ matrix. Select the column with the largest magnitude as the most estimable parameter. Set $k=1$.

2. Construct the matrix $\mathbf{X}_{k}$ by including the $k$ selected columns from $\mathbf{Z}$ that correspond to parameters that have been ranked.

3. Use $\mathbf{X}_{k}$ to predict columns in $\mathbf{Z}$ using ordinary least squares:

$$
\hat{\mathbf{Z}}_{k}=\left(\mathbf{X}_{k}^{T} \mathbf{X}_{k}\right)^{-1} \mathbf{X}_{k}^{T} \mathbf{Z}
$$

and calculate the residual matrix:

$$
\mathbf{R}_{k}=\mathbf{Z}-\hat{\mathbf{Z}}_{k}
$$

4. Calculate the magnitude of each column in $\mathbf{R}_{k}$. The $(k+1)^{\text {th }}$-most estimable parameter corresponds to the column in $\mathbf{R}_{k}$ with the largest magnitude.

5. Put the columns corresponding to the $k+1$ parameters that have been ranked in the matrix $\mathbf{X}_{k}$.

6. Increase the iteration counter $k$ by one and repeat Steps $2-5$, until all parameters are ranked or until it is impossible to perform the least-squares calculation in step 3 due to matrix singularity. 
Table 3. Initial conditions for center-point batch reactor operation at $T=40{ }^{\circ} \mathrm{C}$

\begin{tabular}{lcc}
\hline State variable & Units & Initial condition \\
\hline$C_{S M 1}$ & $\mathrm{M}$ & 1 \\
$C_{D}$ & $\mathrm{M}$ & 0.05 \\
$C_{S M 2}$ & $\mathrm{M}$ & 1.15 \\
$C_{S M 1 D}$ & $\mathrm{M}$ & 0 \\
$C_{P}$ & $\mathrm{M}$ & 0 \\
$C_{H_{2} O}$ & $\mathrm{M}$ & 0.10 \\
$C_{I 1}$ & $\mathrm{M}$ & 0 \\
$C_{I 2}$ & $\mathrm{M}$ & 0 \\
$C_{I 3}$ & $\mathrm{M}$ & 0 \\
$C_{I 4}$ & $\mathrm{M}$ & 0 \\
\hline
\end{tabular}

This article is protected by copyright. All rights reserved. 
Table 4. True values of the kinetic coefficients and equilibrium constant ${ }^{6}$

\begin{tabular}{lccc}
\hline & Units & Value at $40^{\circ} \mathrm{C}$ & $E_{a, i}$ or $\Delta H_{1}\left(\mathrm{~J}^{\mathrm{mol}}{ }^{-1}\right)$ \\
\hline$k_{1 f}$ & $\mathrm{M}^{-1} \mathrm{~s}^{-1}$ & $1.09 \times 10^{-01}$ & $5.00 \times 10^{4}$ \\
$K_{e q}$ & $\mathrm{M}^{-1}$ & 9.33 & $-1.00 \times 10^{4}$ \\
$k_{2}$ & $\mathrm{M}^{-1} \mathrm{~s}^{-1}$ & $3.39 \times 10^{-03}$ & $4.25 \times 10^{4}$ \\
$k_{3}$ & $\mathrm{M}^{-1} \mathrm{~s}^{-1}$ & $1.09 \times 10^{-06}$ & $9.00 \times 10^{4}$ \\
$k_{4}$ & $\mathrm{M}^{-1} \mathrm{~s}^{-1}$ & $1.17 \times 10^{-05}$ & $9.50 \times 10^{4}$ \\
$k_{5}$ & $\mathrm{M}^{-1} \mathrm{~s}^{-1}$ & $1.93 \times 10^{-06}$ & $9.75 \times 10^{4}$ \\
$k_{6}$ & $\mathrm{~s}^{-1}$ & $1.87 \times 10^{-08}$ & $8.00 \times 10^{4}$ \\
\hline
\end{tabular}

This article is protected by copyright. All rights reserved. 
Table 5. Measurement variances used for generating simulated data

\begin{tabular}{lc}
\hline Measured response $(\mathrm{M})$ & $\sigma_{y_{i}}^{2}\left(\mathrm{M}^{2}\right)$ \\
\hline$y_{S M 1}$ & $4.8 \times 10^{-2}$ \\
$y_{D}$ & $2.1 \times 10^{-4}$ \\
$y_{S M 2}$ & $5.2 \times 10^{-2}$ \\
$y_{P}$ & $5.0 \times 10^{-2}$ \\
\hline
\end{tabular}

This article is protected by copyright. All rights reserved. 
Table 6. Lower and upper bounds for the decision variables

\begin{tabular}{ccc}
\hline Decision variables $(\mathrm{M})$ & Lower bound $(\mathrm{M})$ & Upper bound $(\mathrm{M})$ \\
\hline$C_{S M 1_{0}}$ & 0.10 & 1.5 \\
$C_{D_{0}}$ & 0.01 & 0.5 \\
$C_{S M 2_{0}}$ & 0.10 & 1.5 \\
\hline
\end{tabular}

This article is protected by copyright. All rights reserved. 
Table 7. Selection of parameter initial guesses from normal distributions

\begin{tabular}{lcl}
\hline \hline & $\begin{array}{c}\text { Prior Distribution for the } \\
\boldsymbol{j} \text { th parameter }\end{array}$ & $\begin{array}{c}\text { Rules for selection of parameter initial } \\
\text { guess } \hat{\boldsymbol{\theta}}_{\boldsymbol{j} \mathbf{0}}\end{array}$ \\
\hline $\begin{array}{l}\text { Case I: Informative } \\
\text { initial guess }\end{array}$ & $N\left(\mu: \theta_{j}, \sigma: s_{\theta_{j}}=\frac{1}{5} \theta_{j}\right)$ & $\begin{array}{l}\text { Discard any parameter initial guesses } \\
\text { beyond } \theta_{j} \pm 3 s_{\theta_{j}} \text { and select again. }\end{array}$ \\
\hline $\begin{array}{l}\text { Case II: Moderately } \\
\text { informative initial guess }\end{array}$ & $N\left(\mu: \theta_{j}, \sigma: s_{\theta_{j}}=\frac{1}{2} \theta_{j}\right)$ & $\begin{array}{l}\text { Discard any negative parameter initial } \\
\text { guesses and any values beyond } \theta_{j} \pm 3 s_{\theta_{j}} \\
\text { and selected again. }\end{array}$ \\
\hline $\begin{array}{l}\text { Case III: Misinformed } \\
\text { initial guess }\end{array}$ & $N\left(\mu: \theta_{j}, \sigma: s_{\theta_{j}}=\frac{1}{5} \theta_{j}\right)$ & $\begin{array}{l}\text { Select initial guess all from the tails of the } \\
\text { distribution, beyond } \theta_{j} \pm 3 s_{\theta_{j} .} \text { Discard } \\
\text { any negative value and select again. }\end{array}$ \\
\hline
\end{tabular}

This article is protected by copyright. All rights reserved. 